\title{
La culture créative des organisations comme substrat à l'apprentissage créatif
}

Dany Baillargeon, professeur Université de Sherbrooke dany.baillargeon@USherbrooke.ca 


\title{
Résumé
}

La créativité, autant comme acte processuel que comme résultat de ce processus, est un trope fondateur des agences de communication marketing (McFall, 2004, Nixon 2003). Certains avancent même que la créativité serait la raison d'être des agences (Nixon, 2003). Or pour certains, la créativité ne s'apprendrait pas - en témoigne le parcours académique éclectique des créatifs - ou ne s'apprendrait qu'au contact des pairs (Powell, 2006), à travers des dynamiques de production de sens (Drazin, Glynn \& Kazanjian, 1999), des frictions entre les différents corps d'emploi (Hirschman, 1989) ou au contact des clients (Cronin, 2004). Cet article a un triple objectif: 1) d'abord proposer une définition opératoire pour mieux saisir cette culture créative ; 2) l'exemplifier à l'aide d'une étude de cas dans une petite agence de onze personnes 3) mettre en relation la notion de culture créative avec celle d'apprentissage et de transmission du savoir créatif. Il s'en dégage que la performativité de l'ethos créatif des employés comme celle de l'agence participent à maintenir cette culture créative autant qu'elle transmet des formes d'apprentissage de qui est et de ce qui est créatif à travers des dynamiques identitaires, l'éducation à faire auprès des clients et l'utilisation de structures de légitimation interne et externe à l'agence.

Mots clés: Culture créative, culture organisationnelle, apprentissage organisationnel, agence, publicité.

\begin{abstract}
Creativity, both as a procedural act as well as the result of such a process, is a founding trope of marketing communication agencies (McFall, 2004, Nixon 2003). Some even argue that creativity is the raison d'etre of those agencies (Nixon, 2003). But for some, creativity cannot be learned - as shown by the eclectic academic background of creatives - or it could be learned only through being in contact with peers (Powell, 2006), with clients (Cronin, 2004), through the process of creating meaning (Drazin, Glynn \& Kazanjian, 1999), or frictions between different job categories (Hirschman, 1989). The goal of this article is threefold: 1) to propose an operational definition of "creative culture" in order to better understand it; 2) to exemplify it using a case study of a small agency 3) to articulate a relationship between the three related concepts: culture, creative learning and transmission of creative knowledge. The study demonstrates how the performativity of the creative ethos of both employees and agency interact to maintain this creative culture while indicating who and what is creative, through dynamics of identity, educating clients and the use of internal and external structures of legitimation.
\end{abstract}

Keywords: Creative culture, agency, advertising, organizational culture, organizational learning. 
Pour maintenir l'ethos d'une industrie " pourvoyeuse de créativité » (Nixon, 2003) - unique dépositaire de compétences en production de contenu et en réflexivité stratégique -, les agences placent les «créatifs » et la créativité au cœur de leur modèle d'affaires. Or cette créativité, continuellement débattue et faisant l'objet de moult médiations (entre pairs créatifs, entre collègues d'agence, avec les clients et au sein de structures institutionnelles) ne fait pas consensus. Une définition de qui est et de ce qui est créatif est à chaque nouveau projet mise au ballottage, et les créatifs demeurent "aussi bons que leur dernière création" (Hackley \& Kover, 2007). À l'issue de dynamiques de légitimation, performées dans le discours et les actions des membres de l'agence, c'est toute une culture qui est produite au sein des agences, sur fond de culture commerciale (McFall, 2004 ; Sassatelli, 2007) et du culte de la nouveauté, de l'original, de l'innovant (Negus \& Pickering, 2000). Mais quid de cette culture lorsque les régimes de médiation n'impliquent que quelques personnes aux rôles et identités professionnelles floues? Que la culture commerciale n'est pas celle des grandes marques, mais bien de petits annonceurs? Comment, hors des grands circuits institutionnels - concours, associations professionnelles, presse professionnelle - fixant l'ethos du créatif, un employé d'une petite agence de publicité en région, conçoit-il son travail et apprend-il celui-ci ?

Ainsi posons-nous cette recherche sur la culture créative des petites agences de publicité en région, la façon dont elle est maintenue et légitimée et comment, au prisme de facteurs régionaux, elle favorise un apprentissage de ce qu'est la créativité.

\section{Conceptualisation de la culture créative}

\subsection{Vers une définition opératoire de la culture créative des agences de publicité}

Tirant profit des fondements anthropologiques de la culture selon Geertz (1973), la perspective culturaliste des organisations (Alvesson, 2002; Bantz and Pepper, 1993; Eisenberg \& Riley, 2001; Mahy, 2011; Smircich, 1983a, 1983b) part à la recherche de structures signifiantes, ses strates de sens que recèlent les interactions entre les acteurs de l'organisation. Ainsi, la métaphore de la culture organisationnelle permet de saisir un large spectre d'idéologies, de symboles, de mythes, de textes, allant du discours des leaders, aux rituels, langage en usage et mythes ; des textes corporatifs et des narratifs d'organisation au champ complet du non verbal, de la structure d'un stationnement aux structures processuelles du travail (Eisenberg et Riley, 2001). En regard de cette posture, l'organisation est une culture : elle n'est donc pas « une partie du casse-tête, mais le casse-tête lui-même» (Pacanowsky et O'DonnelTrujillo, 1983, p. 146). 
Ce «système de symboles, tissu de conversations, espace mystique, véhicule de complexités au sein duquel les humains deviennent des animaux symboliques, porteurs de sens et en quête de sens » (Mahy, 2011, p. 113), est donc « tissé » par les interactions entre les individus, mais également au contact des choses. «On doit prêter attention au comportement, et cela avec une certaine précision, parce que c'est à travers le flux du comportement - ou, plus précisément, de l'action sociale - que les formes culturelles s'articulent. » (Geertz, 1973, p. 17).

\subsection{Culture de consommation et culture publicitaire}

Appréhender la culture d'une organisation nécessite de saisir le contexte dans lequel elle s'inscrit. En cela, la culture publicitaire est directement inscrite dans la culture du commerce : «La culture commerciale, en ce sens, est utilisé pour saisir les « cultures de commerce »: les significations et les valeurs culturelles adoptées de façon à fixer les conditions d'affaires et le commerce» (Nixon, 2003, p. 35. Nous traduisons). Cette culture du commerce, enclavée dans la culture de consommation (Featherstone, 2007 ; Sassatelli, 2007 ; Sherry et Fischer, 2009), est trop vaste pour être détaillée dans le présent texte. Toutefois, notons que dans une perspective culturelle, la consommation est définie comme le résultat d'une dynamique de production et de consommation :

[...] la consommation ne peut plus être considéré comme un acte innocent, mais dans le cadre des chaînes d'interdépendance et de réseaux qui lient les gens à travers le monde en termes de production, de consommation et également l'accumulation des risques. [...] En somme, la culture de la consommation implique de la connaissance. (Featherstone, 2007, p. xvii, xviii. Nous soulignons et traduisons.)

C'est parce qu'elle implique cette «chaîne d'interdépendances » que la consommation doit être observée comme un phénomène non pas causal d'une stratégie de production, mais bien comme le résultat d'une dynamique de classification et de déclassification, d'interprétation, de circulation de symboles et de production de sens par les consommateurs (Bourdieu, 1977 ; Bourdieu, 1979). Au cœur de l'activité commerciale réside le «culte de la nouveauté » (Nixon, 2003) engendrant le roulement incessant du « plaisir, des désirs frustrés, de l'obsolescence et des nouveaux désirs » cher au marketing (Negus et Pickering, 2000, p. 11).

Ce culte de la nouveauté a placé les créatifs - concepteurs rédacteurs, designer, directeurs artistiques - au centre du modèle d'affaires des agences. Omnivores culturels, les créatifs sont perçus comme les seuls pouvant articuler la relation entre économie et culture, affaires et art (McFall, 2002, 2004) et possédant la sensibilité artistique nécessaire pour interpeller les masses (Hackley et Kover, 2007). Même 
l'organisation physique (bâtiments, organisation de bureaux, espaces ouverts) et structurelle (corps d'emploi) des agences confère aux créatifs un statut particulier : leur reviennent les salaires les plus élevés (Smith et Yang, 2004) et les postes les plus influents (Till et Baack, 2005).

Dans une industrie où les créatifs bâtissent leur carrière n'ont pas en termes verticaux, leur ascension au sein de l'entreprise, mais comme une entreprise de capital personnel (Bourdieu, 1977 ; Nixon, 2003), la question de l'identité et de la légitimation est d'autant plus importante. Les prix, les concours et les autres structures de reconnaissance de cette créativité deviennent par conséquent des entreprises de capital social.

Plus encore, les entreprises dont le produit repose sur la maîtrise de connaissances expertes, comme les agences de publicité, doivent constamment démontrer leurs compétences de façon à réduire l'incertitude et l'insécurité de leurs clients (Powell, 2006). Alvesson $(2001 ; 2004 b)$ avance que la performativité de cette connaissance tient davantage de «l'interprétation et des croyances » que de l'utilisation de réelles connaissances expertes. Devant la difficulté à démontrer l'objective pertinence de leur savoir et l'incapacité de leurs clients à l'apprécier, les travailleurs des entreprises du savoir déploient davantage d'efforts pour développer une image professionnelle et experte, appuyée par une rhétorique autour de la valeur de leur expertise. Au point où, selon Alvesson, l'utilisation du savoir en lui-même n'est plus au cœur du travail.

Cela implique un changement d'emphase: d'une maitrise formelle de la connaissance vers des stratégies de persuasion pour convaincre toutes les parties concernées de la rationalité supérieure de cette l'expertise dont ils peuvent bénéficier. (Alvesson, 2001, p. 872. Nous traduisons)

D'où une prédominance d'activités de contrôle sur la culture et l'idéologie, les valeurs, les idées, les croyances de l'organisation, avec un impact considérable sur l'identité.

Compte tenu du niveau élevé de l'ambiguïté et la fluidité de la vie organisationnelle et des interactions avec les acteurs externes, impliquant une forte dépendance sur les évaluations et les opinions parfois arbitraires de ceux-ci, de nombreux travailleurs du savoir doivent davantage lutter pour la réalisation, l'entretien et le changement progressif de l'identité, par rapport aux travailleurs dont les compétences et les résultats sont plus matériellement ancrés. (Alvesson, 2001, p. 877. Nous traduisons).

De façon à maintenir cette image forte de pourvoyeuse d'un savoir qu'elles seules possèdent - et qui échappe souvent à leurs clients -, les agences exhibent les 
témoins de cette maîtrise : site Internet, livre d'agence, portfolio, prix remportés et clients prestigieux.

\subsection{Saisir la culture créative : une définition opératoire de la culture créative des agences de publicité}

En somme, la culture créative d'une organisation donne un sens aux actions autant qu'elle (re)produit les identités des travailleurs. Chaque performance (Trujillo, 1983) des créatifs, que ce soit en affirmant leur position, en produisant une publicité, en définissant le fonctionnement des agences, constitue une ventriloquie (Cooren, 2010) de cette doxa créative. Pour saisir ce flot évanescent d'actions symboliques, nous proposons une définition opératoire de la culture créative des agences comme :

1. des dynamiques de légitimation de la créativité en regard d'attentes endogènes et exogènes à l'agence,

2. performées à travers l'interaction entre les individus et les choses,

3. qui organisent les rapports de production et de consommation

4. (re)produisant une idéologie variable de la créativité publicitaire.

Certes, une telle conceptualisation ne stabilise en rien cette culture. Ou pour reprendre Geertz, la « condition première de la théorie de la culture est qu'elle n'est pas son propre maître. » (1973, p. 25. Nous traduisons)

Toutefois, elle admet un certain nombre des considérations développées précédemment, en plus de rendre plus maniable la notion de culture (organisationnelle), concept instable, fluide, comme un «vortex» (Martin, 2002) qui aspire celui qui s'aventure à la décrire. D'abord, la culture de la créativité est un processus, donc en rien monolithique, ni stabilisé, ni stratégiquement inoculée, qui émane de la reconnaissance de qui est et de ce qui est créatif. Cette dynamique traverse les êtres (individus et choses), chaque fois que sont performés ces actes de légitimation, que ce soit par le regard des pairs comme celui des agents extérieurs à l'agence (clients, consommateurs). Par les tensions et négociations entourant qui est et ce qui est créatif - donc des définitions opératoires de la créativité -, ces dynamiques participent à organiser le sens des actions et à réitérer les identités professionnelles et organisationnelles. Au contact de ces dynamiques s'apprend le travail d'agence.

\section{Culture créative, petites agences et créativité régionalisée}

Ayant fixé cette définition opératoire, nous la suspendons temporairement, le temps de nous saisir d'une étude de cas nous permettant d'illustrer certains pans de 
la culture créative d'une petite agence de publicité en région et comment elle participe à faire circuler l'apprentissage de qui est et de ce qui est créatif. Nous la réintroduirons par la suite comme outil pour analyser les matériaux empiriques et ainsi en montrer l'opérationnalisation et pointer les moments d'apprentissage.

Cette observation sur le terrain a eu lieu en mars et juillet 2013. Durant trois semaines, nous avons étudié le cas de la Boîte ${ }^{74}$, une agence de onze employés, offrant des services-conseils en communication marketing intégrée - design graphique, stratégie de communication, production numérique - dans une région non métropolitaine, donc hors de Montréal et de la ville de Québec, au Canada. Au moment du terrain, l'agence était composée de deux directrices artistiques, d'une rédactrice-conceptrice/gestionnaire de projets, d'un graphiste/gestionnaire de projets, de quatre programmeurs, dont l'un est aussi coassocié, de deux représentants commerciaux et de l'associé principal. Une ressource comptable à temps partiel se joint à l'équipe par moments ${ }^{75}$.

Par l'entremise d'entrevues individuelles semi-dirigées, de captations vidéo de trois réunions de production, huit réunions de travail de même qu'un questionnaire de vingt-cinq questions, nous avons tenté d'observer quelles étaient les particularités d'une culture créative d'une petite agence de publicité en région (PAPR). Suivant une approche par théorisation ancrée (Corbin et Strauss, 1990, 2010) et dans une perspective constructive interprétative (Charmaz et Belgrave, 2012), selon laquelle une réalité de cette culture est coconstruite par l'interaction avec les acteurs en plus d'observer comment les interactions entre les acteurs et leur environnement produisent cette culture -, les données recueillies ont été compilées dans un logiciel de forage de données qualitatives de façon déterminer des thèmes récurrents qui ont trait à cette culture créative.

Nous ne prétendons pas avoir épuisé l'ensemble des actions productrices de cette culture, et bien que nous ayons privilégié une étude en profondeur d'une culture plutôt qu'une approche macro, - de façon à voir cette culture en action autant qu'en description -, nous pouvons dégager des modes porteurs de circulation de cette culture. Nous avons pu observer trois thèmes porteurs de la culture créative de

\footnotetext{
${ }^{74}$ Conformément à l'entente avec l'agence, toutes références à celle-ci ont été rendues anonymes. Le nom des employés a également été changé.

${ }^{75} \mathrm{La}$ direction artistique voit à la conception visuelle de la publicité ; la rédaction-conception réalise les portions écrites de la publicité en plus de déterminer l'orientation créative d'une campagne (slogan, thème, stratégie); la gestion de projet s'occupe du suivi des étapes de production et de la relation entre les différents employés impliqués dans un projet ; un graphiste, contrairement à un directeur artistique, s'occupe de rendre conformes aux différentes normes d'impression les réalisations des directeurs artistiques en plus de voir au respect des différentes chartes graphiques du client; les programmeurs transfèrent en interfaces numériques les concepts visuels des directrices artistiques; les représentants des ventes développent et maintiennent les relations d'affaires entre des clients et l'agence.
} 
cette agence de publicité, à savoir 1) des dynamiques identitaires modulées par la taille de l'agence ; 2) l'impact d'une «régionalité » sur une éducation à faire au client; 3) des légitimations alternatives propres à cette régionalité. Ces trois thèmes recèlent autant de modes de transmission et d'apprentissage de la culture créative.

\subsection{Faire plus petit : les identités fragmentées}

Travailler dans une petite agence de douze employés suppose une structure hiérarchique moins définie, avec des descriptions de tâches parfois floues. Chez la Boîte, cette perméabilité des rôles constituerait une «force » et est empreinte d'un esprit de collaboration, « comme une grande famille », où chacun doit «mettre la main à la pâte $»^{76}$. Toutefois, alors que l'on pourrait croire que cette liberté hiérarchique dissout les tensions types entre « créatifs » et « vestons » (Hackley \& Kover, 2007), elles reviennent autrement.

Marie, qui occupe à la fois le rôle de gestionnaire et de conceptrice-rédactrice, se trouve écartelée entre produire la créativité et la gérer, le second l'emportant souvent sur le premier. Elle dit d'ailleurs ne pas avoir le recul nécessaire pour se sentir réellement inspirée, le brief créatif ${ }^{77}$ étant une étape importante de prise de distance par rapport à la commande du client.

Parfois des projets de création sont mis de côté parce qu'il faut répondre aux clients et eux s'attendent à ce que tu répondes dans la seconde. [...] Sinon [le double statut] se gère, mais dans un monde idéal ce sont des postes qui seraient séparés. Je n'aurais pas à m'auto-briefer.

Plus loin dans l'entrevue, elle dit d'ailleurs que ce qui l'influence le plus son processus créatif, c'est la réponse des clients, alors que pour ces collègues directrices artistiques, donc plus campées dans leurs rôles créatifs, les collègues de l'agence ont davantage de poids. Elle se voit souvent contrainte à ramener le créatif dans la direction du client, comme lors de cette session de remue-méninge (brainstorm) pour produire les panneaux réclame d'un nouveau client: «C'est ben beau faire une campagne billboard, mais si on ne répond pas au besoin du client, parce qu'on a voulu juste drôle, pis l'fun, pis beau... on n'a pas réussi notre job ». Par cette défense des intérêts du client, elle réaffirme un élément de la culture : celle du client avant celle de la performance esthétique ou ludique.

Ces identités distendues rendent, par moments, le rapport à la tâche comme « schizophrène », selon Luc, développeur web, responsable du volet multimédia, et

\footnotetext{
${ }^{76}$ Thèmes régulièrement cités par les participants.

${ }^{77}$ Le brief créatif est la transformation des besoins du client en instructions inspirantes pour l'équipe de production (rédacteur, concepteur, graphiste, programmeurs).
} 
coassocié : « Je butine un peu partout alors ça rend la planification et le respect des échéanciers un tantinet plus complexe». Katherine, directrice artistique, soutient d'ailleurs que ce chevauchement des tâches plombe le processus créatif :

[...] la personne en rédaction est aussi chargée de projet et est ici trois jours et demi par semaine. Alors elle ne fait pas partie autant que l'on voudrait du processus créatif. Quand elle met son input, c'est important, mais en même temps elle n'est pas tout le temps là alors c'est quand même difficile.

Cette frustration émane en partie du fait que les deux directrices artistiques soutiennent davantage une identité créative et ont le sentiment d'être "assises carrément dans la chaise des créatifs » (Véronique, directrice artistique). En fait non seulement sont-elles assises dans la chaise de créatifs, mais elles partagent littéralement la même table de travail, contrairement à tous leurs collègues qui ont des bureaux individuels. Et elles semblent les plus promptes à défendre cette position : " Je ne crois pas qu'on fait autre chose et qu'on est intéressée à faire autre chose. Je crois que c'est une question aussi de volonté de ce que tu veux faire.» (Katherine, directrice artistique).

Fait notable, bien que les deux directrices artistiques défendent leur ethos créatif, cette identité ne trouve pas le même écho chez les autres employés, qui préfèrent voir la créativité comme émanant de l'ensemble, « qu'elle vient de tout le monde ».

- «Une personne va prendre le lead du projet côté design, mais ce ne sera pas SON projet. » (Pascal, gestionnaire de projets).

- «Non, on essaie de ne pas faire cette distinction-là parce que la bonne solution et la bonne idée peuvent venir de n'importe qui.» (Luc, développeur web, responsable de la production multimédia et coassocié).

La propriété multiple d'un projet créatif opère à travers des réunions de production où sont débattus les tenants et aboutissants de la commande d'un client. Toutefois, jusqu'à tout récemment, cette mise en commun se faisait de façon improvisée. Or une structure de partage et un système de gestion de projets, importés par Marie qui travaillait dans une agence de Montréal, permettent de mieux faire circuler les enjeux des projets $^{78}$.

Aussi, peut-être parce que depuis 6-7 mois on apporte des changements dans les procédures, parce qu'il n'y en avait pas avant. On a tranquillement donné notre

\footnotetext{
${ }^{78} \mathrm{Au}$ moment de notre passage, cette standardisation de la gestion des projets constituait un important changement et au cœur de nombreuses discussions.
} 
point de vue pour améliorer les choses, moi et Pascal, aussi qui était là avant moi, avait un peu la même réflexion. Ensemble on a réussi à imposer notre façon de faire ce qui fait en sorte qu'on roule mieux. [...] Il y avait un document du parfait chargé de projet qui avait été fait, qui donnait un peu leurs façons de faire du départ, les grandes lignes. Mais comme y'avait rien dans l'appliqué, y'avait les grandes lignes sur comment il faut que ça fonctionne, mais y'avait pas de moyens.

Cette standardisation constitue dès lors un vecteur important de la culture : transmettre une compréhension et un savoir sur les projets en cours et les défis qui attendent l'équipe. Avec cette standardisation s'organise les rôles et s'organise la culture créative :

Elles [les procédures] tendent à beaucoup changer. De un, parce que justement, on a des nouveaux membres qui se sont greffés à l'équipe. [...] De l'avoir concrétisé vraiment pour que, justement, tout le monde travaille sur le même pied. Ce n'était pas possible avant. Avant que l'équipe soit relativement assez grosse pour que ça vaille la peine d'implanter quelque chose. Donc ça, j'ai comme l'impression qu'on devient plus une agence avec les rôles mieux établis.

\subsection{Apprendre à éduquer}

Bien que les employés de la Boîte souscrivent à cette «créativité avec un but » ${ }^{79}$, de sorte que "l'imagination et la créativité font partie de notre $\mathrm{ADN}$ » ${ }^{80}$, il semble que les clients que dessert la Boîte ne témoignent pas de cette créativité. Ce qui n'est pas sans impact sur la perception du travail créatif: alors que cinq des onze employés ont répondu qu'ils considéraient la Boîte comme étant " très créative », seulement deux voient leurs clients comme de dignes représentants de cette créativité.

Ce paradoxe tiendrait d'un manque de compétence chez leur client pour bien saisir la pertinence d'une stratégie de communication créative et d'y adhérer. De fait, tous les participants ont parlé, d'une manière ou d'une autre, du «manque de sensibilité visuelle », «d'éducation à faire », « de clients qui ne comprennent pas ce que l'on fait ».

Souvent en région, c'est un peu là où le bât blesse, parce que les clients comprennent moins bien, l'éducation sur ce que l'on fait est encore à faire, versus d'autres clients de Montréal qui ont travaillé avec plusieurs agences. [...]

\footnotetext{
${ }^{79}$ Autocollant promotionnel pour l'Agence, apposé sur l'ordinateur d'une directrice artistique

${ }^{80}$ Document de l'agence.
} 
Beaucoup ne savent pas ce qu'est une agence de communication. (Marie, gestionnaire de projets, conceptrice-rédactrice).

Si tu es prêt à travailler dans une grosse agence à Montréal, c'est parce que tu connais l'impact que le visuel peut avoir. Alors peut-être que oui, il y a peut-être une sensibilité visuelle qu'il n'y a pas ici, ou à peu près pas. (Véronique, directrice artistique).

Selon les participants, plusieurs clients considèrent l'agence comme productrice de pièces publicitaires, son expertise n'étant prisée que pour ses compétences techniques : produire un dépliant, faire un logo, programmer un site Internet, etc. Dès que l'agence tente d'y apporter une réflexion stratégique, sa pertinence échappe au client.

C'est beaucoup d'éduquer c'est quoi une agence de communication, qu'est-ce qu'on fait et une des réponses que l'on a quand on soumissionne pour des projets c'est «c'est dont ben cher et dont ben du temps » [...] Mais c'est ça, il faut réfléchir à ton produit, tes besoins, faire des recherches. C'est une notion qui n'est pas connue. (Pascal, gestionnaire de projets, graphiste).

Cette éducation doit également se faire pour défendre l'impact d'une communication qui transcende le simple outil. Parlant de la façon de concevoir des sites Internet de son ancien employeur, Luc fait justement cette distinction entre l'outil et la stratégie :

On livrait quelque chose de vide. Un header, un footer, un fond, un espace pour écrire et un outil au client pour permettre d'écrire leurs textes. Ça peut être bon pour une certaine clientèle, mais en bout de ligne si tu veux quelque chose de performant et qui se démarque, ce n'est pas assez. Ça prend des réflexions. [...] On a des clients qui nous engagent comme plus serviceconseil, les aider à la communication, les guider. Et il y des clients qui nous engagent comme purement des exécutants. On voit les deux. Il y a des clients qui nous arrivent avec une maquette toute faite. Il veut son site comme ça, et dans trois semaines.

La Boîte doit même défendre la pertinence pour un annonceur de s'adresser à une cible, alors que le client a tendance à se projeter dans celle-ci : « [...] on est souvent dans cette tension-là, d'essayer de dire “oui, mais ce n'est pas à toi à qui ça s'adresse. Notre client final ce sont tes clients parce qu'il faut que ta clientèle cible soit capable d'y trouver son compte" ». Ce qui expliquerait, selon Luc, le « pourquoi des gérants et concessionnaires [automobiles] qui se mettent dans leurs publicités en se trouvant eux-mêmes plus importants qu'ils le sont ». 
Même la pertinence d'aider un client à se distinguer est débattue. En l'absence d'une concurrence féroce, autre conséquence du contexte régional, certains clients ont tendance à émuler les communications de leurs compétiteurs plutôt que de tenter l'aventure de la distinction.

Par exemple, si tu es une compagnie de construction à Montréal, la compétition est beaucoup plus forte, tu sais que tu dois vraiment mettre de l'emphase sur la pub, ton logo, comment tu te présentes. (Véronique, directrice artistique)

Expliquant pourquoi un projet de site Internet en était rendu à sa quatrième mouture, Luc parle également de cette émulation, comme d'un refuge quand un client ne comprend pas l'utilité « d'aller plus loin que ce qui marche ».

On avait l'impression qu'il était ouvert à aller ailleurs pour finalement se rendre compte qu'il nous ramenait toujours au site de ses compétiteurs. Il a fini par nous dire qu'il ne voulait pas se démarquer de ses compétiteurs.

Dans le cas de la Boîte, le fait que plusieurs clients approchent l'agence que pour produire une pièce publicitaire $-80 \%$ de la clientèle selon un des coassociés contribuerait justement à ne pas permettre à cette créativité d'être développée à son plein potentiel.

[...] il faut être réaliste aussi. Se faire dire « tu peux aller loin, loin, loin ». Oui je sais, mais quand c'est une pub d'un pouce par un pouce en noir et blanc, là, ce n'est pas le moment d'être créatif. (Katherine, directrice artistique)

D'autant que l'accès à un degré de sophistication chez les clients semble corrélatif à la taille de l'entreprise. Dans un contexte régional où l'on compte un nombre moins important de grandes entreprises, l'impact de cette «éducation à faire » semble plus important :

[...] quand tu arrives avec des plus grosses [entreprises] qui ont des départements de marketing, elles réalisent l'importance de la mise en marché, de la communication et du marketing [...] Oui, on a peut-être moins accès facilement à ce genre d'entreprise là, alors ça peut avoir un impact sur le fait qu'on soit en région. (Luc)

Pour pallier ce manque de connaissance des processus créatifs de même que des impacts souhaités d'une campagne de communication, la Boîte a conçu des documents pour mieux éduquer leurs clients: formulaire de prise de besoin, document d'agence qui explique « une stratégie, qu'est-ce que ça mange en hiver ? » 
et que « faire du design, c'est plus que des points et des lignes bien alignés ${ }^{81}$. Qui plus est, la standardisation des processus, dont il a été question précédemment, a permis de mieux éduquer le client sur la valeur de cette stratégie :

Je pense que, avec le Task manager ${ }^{82}$, c'est facile d'expliquer les heures, puis de justifier « bon ben, voici le temps qui a été mis ». C'est une question, je pense, de transparence, puis d'expliquer au client qu'est-ce qui en est. Parce que les gens ne saisissent pas toujours le temps que ça peut prendre.

Donc, transmettre la valeur du travail au client, mais également éduquer l'équipe à la nécessaire rentabilité du processus créatif :

Puis depuis quelques mois, je trouve ça bien, parce que je n'ai plus besoin vraiment de répéter [l'importance du contrôle des heures]. C'est eux, ils ont leur brief, ils ont un temps à respecter. C'est important là, le côté rentabilité de l'entreprise, sinon, on n'existera plus.

\subsection{Structures de légitimations alternatives}

À Montréal, mes amis qui sont chez Orange Tango, il y en a qui sont partout et puis eux ils gagnent des prix et je ne trouve pas que j'ai moins de talent qu'eux. Je ne suis juste peut-être pas dans la bonne ville ou dans la bonne agence. (Katherine, directrice artistique).

Comme nous l'avons souligné précédemment, les structures légitimantes exogènes que sont les prix, les concours, les associations professionnelles, tendent à stabiliser une certaine définition de ce qui est créatif en plus de soutenir l'identité créative de l'organisation. Or les agences régionales n'accèdent pas aux mêmes mécanismes de validation que les grandes agences : on remarque une absence manifeste des agences régionales dans la presse professionnelle ${ }^{83}$, dans les associations professionnelles ${ }^{84}$

\footnotetext{
${ }^{81}$ Document d'agence

${ }^{82}$ Logiciel de gestion des tâches et des heures accordées à chaque projet

${ }^{83}$ Par exemple, des 553 articles publiés entre 2000 et 2012 dans la catégorie « agences » sur le portail du marketing, de la publicité et des communications au Québec Infopresse.com, aucun ne porte sur une agence autre que montréalaise.

${ }^{84}$ L'Association des agences de publicité du Québec (AAPQ), dont la mission est d'être « à l'affût des enjeux et des tendances de l'industrie de la communication, [de] promouvoir, défendre et [de] représenter les intérêts de ses membres et d'outiller les décideurs d'aujourd'hui et la relève de demain afin de relever les défis de l'évolution de l'industrie » compte seulement six membres, sur 70, qui ont pignon sur rue hors de Montréal ou de Québec.
} 
et dans les concours de l'industrie de la publicite ${ }^{85}$. La Boîte ne fait pas exception. Elle doit malgré tout soutenir son ethos de pourvoyeuse de créativité, qu'elle assoit sur des structures de légitimation endogène et exogène, propres à l'agence et à son contexte régional.

À l'interne, cette créativité est débattue comme il se doit, par une rhétorique sur la valeur d'une idée.

[...] si tu es capable d'assumer ce que tu as fait et de l'expliquer et de le justifier solidement et concrètement et nous convaincre que c'est la meilleure idée, c'est parce que ton idée est bonne. (Luc, coassocié et responsable de la production multimédia)

Un moment probant pour la circulation de cette rhétorique, la refonte des documents de présentation de l'agence et du site Internet a constitué un lieu d'échange sur l'évolution de la culture créative de l'agence.

Je pense que ça démontre aussi notre personnalité, puis qui on est. J'ai adoré ce qu'elles [les directrices artistiques] m'ont présenté les dernières semaines. Enfin, on est arrivé un petit peu à ce que j'avais en tête depuis le jour un. [...] Puis là, on s'est assis, puis on a essayé de se redéfinir aussi beaucoup. C'est un travail qui est dur, de travailler sur soi-même là, puis [sur] ces documents, puis le faire arriver à travers les clients aussi.

Bien que « chacun ait sa propre définition de la créativité ", selon Sébastien, président de l'agence, les réunions de production, où sont partagés et discutés les projets en cours, tendent à stabiliser cette définition. De façon informelle, les employés de la Boîte semblent partager beaucoup de trouvailles, entre autres par un logiciel de partage, même si le "partage, c'est ce qui fait défaut ici », avance Luc, directeur de la production multimédia et coassocié. Depuis peu, des «ateliers créatifs » ont été mis sur pied pour que s'échangent réflexions sur la créativité, pratiques d'excellence, résultats de concours, etc. Selon le questionnaire, c'est toutefois à travers les réunions de projet que cette culture créative se diffuserait le plus.

En regard de la légitimation exogène, un facteur intéressant point : la proximité.

\footnotetext{
${ }^{85}$ Des 193 prix remis entre 2010-2012 lors des concours Créa et Strat, destinés aux campagnes québécoises, aucune agence hors Montréal ou hors de la ville de Québec n'a remporté de prix. De plus, 181 prix ont été accordés à de grandes agences (50 employés et plus), un prix à une moyenne agence (20 à 49 employés), et sept prix à de petites ou très petites agences ( 1 à 19 employés). Encore une fois, nous observons une surreprésentation des grandes agences qui pourtant ne comptent que pour $27,5 \%$ des employeurs de l'industrie publicitaire.
} 
Si on prend [ville], ça reste que c'est plus petit donc tout le monde se connaît, il y a une espèce de « lui a tel client, j'ai tel client» (Pascal, gestionnaire de projet et graphiste).

La proximité entre les différents acteurs du milieu - agence comme client - fait en sorte qu'une légitimation opère dans qui réussit à obtenir les clients ayant une plus forte visibilité ou étant reconnus comme plus « ouverts créativement».

Ici, je commence à le voir, « OK lui a travaillé là, tel client est rendu ici, avant il était chez lui ». Ça se voit, ça se sent, c'est un peu ça le challenge d'une petite agence en région, c'est d'aller chercher les clients. [...] C'est vrai à Montréal aussi que les agences se connaissent, mais il y en a tellement que ça se diffuse un peu (Véronique, directrice artistique).

Certes, d'aucuns pourraient arguer que de connaître les mouvements de compte est aussi un (important) exercice de légitimation chez les grandes agences métropolitaines. Toutefois, dans le cas d'une entreprise régionale, cette valse des clients n'est pas médiée par la presse spécialisée, mais davantage le fruit de discussions informelles, en forme de sociabilité entrepreneuriale ${ }^{86}$.

Cette même proximité vient également jouer sur la reconnaissance du caractère original des productions de la Boîte. De fait, selon Katherine et corroborée par Pascal, la Boîte a établi en quelque sorte une signature qu'on lui attribue :

Je pense qu'on se démarque visuellement à [Ville]. [...] C'est ça qui nous démarque beaucoup. On parle de [client], on a souvent le commentaire «wow c'est le fun, on le voit partout » [...] Premièrement parce que visuellement c'est un peu différent de ce que l'on voit et il y a un résultat. Le but c'est de rendre nos clients les plus visibles possible et ça fonctionne. (Pascal)

D'ailleurs, ce client «dont tout le monde parle »- et dont les panneaux réclame tapissent la ville - semble être en effet le client qui est le plus fidèle témoin du travail de la Boîte : tous les participants l'ont cité comme un « digne représentant de votre créativité ». Toutefois, il semblerait que ce soit davantage parce qu'il est « partout » que parce qu'il est vraiment créatif.

De fait, les deux directrices de création semblent trouver ce client «quelconque » ou "davantage de l'exécution », même si elles savent que leurs collègues en sont fiers. Ainsi, qu'on parle d'un client pour sa présence médiatique et parce qu'il admet

${ }^{86}$ Anecdote révélatrice : alors que nous captions une rencontre avec un client dans le domaine de l'éducation sportive, la mère de ce dernier, qui possède une boutique de vêtements, faisait son entrée dans l'agence quelques minutes plus tard. 
« un minimum d'unité graphique » serait suffisant pour soutenir l'ethos créatif de la Boîte. Cette définition de ce qui est créatif est d'ailleurs réitérée dans un échange entre le patron de la Boîte et Véronique concernant les clients considérés comme inspirants :

Véronique : [...] on a $50 \%$ que c'est alimentaire que c'est moins créatif que c'est de la répétition. Aussitôt que c'est répétitif pour moi, c'est moins créatif.

Sébastien : Ouais, mais il a une créativité dans ça quand même, on l'a parti au départ et on continue la ligne.

Ainsi, la légitimation exogène est à la fois construite autour d'une réflexion stratégique qui «continue la ligne » et qui serait perçue publiquement. Qui plus est, cette direction stratégique convoyant une uniformité esthétique est le seul rempart à un «style régional»: «C'est le premier niveau l'idée du patron narcissique qui dit "ah je suis un plombier alors j'ai une photo de moi habillé en plombier qui dit je suis un plombier" ».

Ce qui conduit les professionnels à s'expliquer mutuellement leur créativité de sorte à pouvoir la débattre auprès du client, comme dans cet échange entre les deux directrices artistiques et Marie qui tentent de trouver un concept pour un nouveau client dans le domaine de la santé. Alors que Marie lui présente des concepts « droits au but », Valérie soutient que l'idée répond aux objectifs, mais pêche par excès de convention. Katherine nuance en soutenant qu'au moins, l'idée n'est pas une surenchère dénotative.

Véronique : C'est tranquille.

Katherine : En même temps, ce qui est l'fun, c'est pas à 100 \% «nous faisons des orthèses $»$.

Marie : C'est tranquille, mais ils ne sont pas connus.

Puis plus tard dans l'échange :

Véronique : C'est un peu du déjà vu. C'est correct, ça fait le job. Ce n'est pas négatif non plus. On peut jouer cette carte-là. C'est safe pour que les gens comprennent. C'est aussi valable si on va là-dedans.

Marie : Le gars n'est pas connu. Le service en soi est un peu flou pour une bonne partie de la population. Il veut s'attirer de la clientèle. On ne veut pas être trop plate. Mais faut que le message passe.

Cette tension définitoire entre un premier degré trop dénotatif se manifestera lorsque Marie rencontrera le client. 
Client : Sur un billboard, est-ce qu'on pourrait montrer un ongle avec un laser et écrire « Le laser pour vos ongles »?

Visiblement peu enthousiaste à cette suggestion, Hubert, le responsable de compte, changera le sujet après un long silence.

\section{Analyse et conclusion : Opérer la culture créative. Apprendre par la culture}

Nous le voyons, la culture créative d'une petite agence de communication marketing en région est sous influence de son contexte régional autant qu'elle émerge de ce qui est fait de ce qui est dit à propos de la doxa créative. Plus encore, à travers ce qui est cultivé s'apprend un certain nombre de savoirs organisationnels et professionnels. D'autant que «la formation scolaire de même que l'affiliation professionnelle constituent des ressources vitales dans le soutien de l'identité [professionnelle], mais en même temps, celles-ci sont constamment en proie au scepticisme et à des remises en cause » (Alvesson, 2004b, p. 218. Nous traduisons). « Ça peut s'apprendre, les grandes lignes, ça peut s'apprendre là, mais ça ne veut pas dire que tu vas les appliquer dans un milieu qui n'est pas contrôlé comme l'école» (Stéphane, représentant commercial).

En reprenant la définition opératoire proposée plus haut, nous pouvons mieux saisir les éléments qui organisent la culture créative de la Boîte et surtout, comment un apprentissage de l'expertise créative est constamment réitéré dans les actions sociales de cette culture.

D'abord, en ce qui concerne les dynamiques de légitimation de la créativité en regard d'attentes endogènes et exogènes à l'agence. Nous remarquons que les identités au sein de la Boîte semblent distendues. De fait, la petitesse de la Boîte, la proximité des rapports entre les différents employés de même qu'une défense d'une créativité souhaitée face à une créativité réaliste imposée par des clients moins sophistiqués, font en sorte que qui est et ce qui est créatif tend à disparaître dans l'organisation. Toutefois, les rôles et tâches des designers, des gestionnaires de projet, des programmeurs des vendeurs tendent à être mieux circonscrits à travers les processus de standardisation, où sont réaffirmés. Pour les employés de la Boîte, ces processus ont permis de transmettre un apprentissage sur comment fonctionne une vraie agence : " "Regardez, c'est ça un processus". En théorie, quand tu as un client, tu as un brief, après ça tu fais tes recherches, des esquisses, des maquettes. » (Katherine, directrice artistique).

La culture créative de la Boîte n'est pas tant le résultat de tensions fortes entre créatifs et gestionnaires - les deux identités étant parfois amalgamées -, mais bien de sa capacité à défendre, auprès de clients à éduquer, de la pertinence de cette créativité, faisant s'estomper l'identité créative d'une personne au profit d'une 
identité créative collective. Nécessaire processus que cette légitimation endogène unifiée lorsque la légitimation exogène semble faire défaut. En l'absence d'institution légitimante - prix, concours, presse professionnelle -, les créatifs d'agence en région semblent se rabattre sur des vecteurs alternatifs: la forte présence médiatique d'un client «dont tout le monde parle»; l'impression qu'un travail de réflexion créative semble émaner d'une stratégie «graphiquement unifiée $»$.

Cette créativité souhaitée est performée dans l'interaction entre les individus et les choses dans la défense d'une rhétorique sur valeur d'une idée. Ainsi, réunions de production, logiciel de partage d'idées, discussions informelles et formelles autour de la créativité sont autant de remémoration organisationnelle (Grosjean et Bonneville, 2009), moments où une idéologie sur la créativité est véhiculée, appelée, (re)construite : « des espaces où les savoirs, les routines, les expériences, les usages, les expertises peuvent circuler, se reconstruire, soutenir la résolution de problèmes, définir de nouvelles pistes de travail, etc. » (Grosjean, 2011, p. 37). L'idée « d'aller plus loin » y est rappelée, mais des objets comme le nouveau processus de gestion de projet avec ses feuilles de production, son gestionnaire de tâche - où temps alloués au projet et budget sont inscrits - viennent circonscrire les combats à mener et rappeler que la culture créative est enchâssée dans la culture du commerce.

Les rapports de production et de consommation sont sous-tendus par des déterminants que sont l'éducation des annonceurs régionaux, leur réticence à investir dans "leur notoriété, tout le non-verbal au fond, le "non-monayable" " (Luc). Ce manque de sophistication (Koslow et al., 2006) vient conditionner ce qui est attendu, confinant parfois la Boîte à un simple exécutant. Ainsi, ce que produit la Boîte et consomme le client empêche «d'aller plus loin » parce que c'est « ça que le client veut » et que «ça sert à rien de se battre ». Alvesson $(2003,2004 \mathrm{~b})$ dirait que l'objectivité de la connaissance experte - un client sait que maîtriser un logiciel de design, concevoir un site internet ou produire un panneau-réclame demande une expertise qu'il n'a pas - prend le pas sur la réelle connaissance experte. Pis encore, faute d'une compétitivité entre les annonceurs, une agence en région doit lutter contre l'émulation à la concurrence, une rhétorique des clients qui ne voient pas l'intérêt de faire différent, puisque ça marche pour mon concurrent. Les rapports de production et de consommation en région obligent les professionnels à apprendre à apprendre : s'expliquer leur travail pour ensuite l'expliquer au client.

In fine, nous avons exposé un certain nombre de facteurs (re)produisant une idéologie variable de la créativité publicitaire. Ainsi, la culture créative d'une agence de publicité en région semble être le fruit d'une ambivalence des rôles créatifs, qui est rapatriée dans la défense de ce qui devrait être créatif, mais soumis à une tension régionale, dont les impératifs sont ceux d'une concurrence moins forte et de clients à éduquer. 
À travers ce qui est cultivé en regard de la créativité, les employés de la Boite apprennent des conventions d'une culture professionnelle à partir des «manières de faire partagées », des routines et théories-en-usage singulières à partir des "manières de faire admises" et, enfin, des accords pragmatiques qui montrent comment des praticiennes s'« arrangent» au quotidien avec certaines contraintes institutionnelles ou pressions sociales à partir des «manières de faire contestées » (Morissette, 2011, p. 19).

\section{Bibliographie}

Alvesson, M. (2001). Knowledge work: Ambiguity, image and identity. Human relations, 54(7), 863-886. doi:10.1177/0018726701547004

Alvesson, M. (2002). Understanding organizational culture. Sage Publications Ltd.

Alvesson, M. (2004a). Organizational culture and discourse. In D. Grant, C. Hardy, C. Oswick, \& L. Putnam (Eds.), The Sage handbook of organizational discourse (pp. 317-335). London: Sage Publications Ltd.

Alvesson, M. (2004b). Knowledge work and knowledge-intensive firms. USA: Oxford University Press.

Bantz, C. R., \& Pepper, G. L. (1993). Understanding organizations: Interpreting organizational communication cultures. South California: University of South Carolina Press Columbia.

Bourdieu, P. (1977). La production de la croyance. arss, 13(1), 3-43. doi:10.3406/arss.1977.3493

Bourdieu, P. (1979). Distinction (La). Paris: Minuit.

Charmaz, K., \& Belgrave, L. L. (2012). Qualitative interviewing and Grounded Theory Analysis. In J. Gubrium, F. \& J. Holstein, A. (Eds.), Handbook of interview research (pp. 347-367). Thousand Oaks: Sage Publications.

Cooren, F. (2010). Ventriloquie, performativité et communication. Ou comment faiton parler les choses. Réseaux, 163(5), 33-54. doi:10.3917/res.163.0033

Corbin, J. M., \& Strauss, A. (1990). Grounded theory research: Procedures, canons, and evaluative criteria. Qualitative sociology, 13(1), 3-21. Page obtenue de http://zfs-online.ub.uni-bielefeld.de/index.php/zfs/article/viewFile/2741/2278

Cronin, A. M. (2004). Regimes of mediation: advertising practitioners as cultural intermediaries? Consumption Markets \& Culture, 7(4), 349-369. doi:10.1080/1025386042000316315 
Drazin, R., Glynn, M. A., \& Kazanjian, R. K. (1999). Multilevel Theorizing about Creativity in Organizations: A Sensemaking Perspective. The Academy of Management Review, 24(2), 286-307. Page obtenue de http://www.jstor.org/stable/259083

Eisenberg, E. M., \& Riley, P. (2001). Organizational Culture. In F. M. Jablin \& L. Putnam (Eds.), The new handbook of organizational communication: Advances in theory, research, and methods (pp. 291-322). Thousand Oaks: Sage Publications, Inc.

Featherstone, M. (2007). Consumer Culture and Postmodernism (2e ed.). California: Sage Publications.

Geertz, C. (1973). The interpretation of cultures: Selected essays (5043). New York: Basic books.

Glaser, B. G., \& Strauss, A. L. (2010). La découverte de la théorie ancrée: stratégies pour la recherche qualitative (iBook ed.). Paris: Armand Colin.

Glaser, B. G. (1978). Theoretical sensitivity: Advances in the methodology of grounded theory (2). Mill Valley, CA: Sociology Press.

Grosjean, S., \& Bonneville, L. (2009). Saisir le processus de remémoration organisationnelle des actants humains et non humains au cœur du processus. Revue d'anthropologie des connaissances, 3(2), 317-347. doi: $10.3917 /$ rac. 007.0317

Grosjean, S. (2011). Actualisation et «mise en scène» de connaissances organisationnelles: Ethnographie des réunions de travail. Recherche Qualitative, $30(1), 60-87$.

Guillemette, F. (2006). L'approche de la Grounded Theory; pour innover. Recherches qualitatives, 26(1), 32-50. Page obtenue de http://www.recherchequalitative.qc.ca/numero26(1)/fguillemette_ch.pdf

Hackley, C., \& Kover, A. J. (2007). The trouble with creatives: negotiating creative identity in advertising agencies. International Journal of Advertising, 26(1), 63 -78 .

Hirschman, E. C. (1989). Role-based models of advertising creation and production. Journal of Advertising, 18(4), 42-53. Page obtenue de http://www.jstor.org/stable/4188739

Koslow, S., Sasser, S. L., \& Riordan, E. A. (2003). What is creative to whom and why? Perceptions in advertising agencies. Journal of Advertising Research, 43(01), 96-110. Page de http://journals.cambridge.org/abstract_S0021849903030113 
Koslow, S., Sasser, S. L., \& Riordan, E. A.. (2006). Do marketers get the advertising they need or the advertising they deserve? Agency views of how clients influence creativity. Journal of Advertising, 35(3), 81-101. Page obtenue de http://mesharpe.metapress.com/index/CP6U149400705236.pdf

Mahy, I. (2011). Les approches symboliques et culturelles. In S. Grosjean \& L. Bonneville (Eds.), La communication organisationnelle, approches, processus et enjeux (pp. 111-139). Montréal: Chenelière Éducation.

Martin, J. (2002). Organizational culture: Mapping the terrain. London: Sage Publications, Inc.

McFall, L. (2002). Advertising, persuasion and the culture/economy dualism. In Cultural Economy: Cultural Analysis and Commercial Life (pp. 148-165). London: Sage.

McFall, L. (2004). Advertising: a cultural economy. London: Sage.

Morrissette, J. (2011). Vers un cadre d'analyse interactionniste des pratiques professionnelles. Recherche Qualitative, 30(1), 60-87.

Negus, K., \& Pickering, M. (2000). Creativity and cultural production. International Journal of Cultural Policy, 6(2), 259-282. doi:10.1080/10286630009358124

Nixon, S. (2003). Advertising Cultures: Gender, Commerce, Creativity. London: Sage Publications Ltd.

Pacanowsky, M. E., \& O'Donnell-Trujillo, N. (1983). Organizational communication as cultural performance. Communications Monographs, 50(2), 126-147. doi:10.1080/03637758309390158

Pettigrew, A. M. (1979). On studying organizational cultures. Administrative science quarterly, 24(4), 570-581. Page obtenue de http://www.jstor.org/stable/10.2307/2392363

Powell, S. M. (2006). Creativity and the corporate brand within small to medium sized creative organisations. Ph.D. University of Strathclyde, Glasgow.

Sassatelli, R. (2007). Consumer culture: History, theory and politics. London: Sage Publications Ltd.

Sherry, J. F., \& Fischer, E. (2009). Explorations in Consumer Culture Theory (Routledge Interpretive Marketing Research). New York: Routledge.

Smircich, L. (1983a). Studying Organizations as Cultures. In G. Morgan (Ed.), Beyond method: Strategies for social research (pp. 160-172). Beverly Hills, CA: Sage Publications, Inc. 
Smircich, L. (1983b). Concepts of Culture and Organizational Analysis. Administrative Science Quarterly, 28(3), 339-358. doi:Article

Smith, R. E., \& Yang, X. (2004). Toward a general theory of creativity in advertising: Examining the role of divergence. Marketing Theory, 4(1-2), 31. Page obtenue de http://mtq.sagepub.com/content/4/1-2/31.short

Till, B. D., \& Baack, D. W. (2005). Recall and Persuasion: does creative advertising matter? Journal of Advertising, 34(3), 47-57. Page obtenue de http://mesharpe.metapress.com/index/BB29AM52UQWLR1W9.pdf

Trujillo, N. (1983). "Performing" Mintzberg's roles: The nature of managerial communication. In L. Putnam \& M. E. Pacanowsky (Eds.), Communication and organizations, an interpretive approach (pp. 73-97). Newbury Park, CA: Sage Publications, Inc. 